\title{
Chemical Bond Activation Observed with an X-ray
}

\section{Laser}

Martin Beye $,{ }^{*}, a, b, c$ Henrik Öberg, ${ }^{d}$ Hongliang Xin,${ }^{e}$ Georgi L. Dakovski, ${ }^{f}$ Martina Dell'Angela,${ }^{g}$ Alexander Föhlisch,${ }^{a, h}$ Jörgen Gladh, ${ }^{d}$ Markus Hantschmann, ${ }^{a}$ Florian Hieke, ${ }^{g}$ Sarp Kaya,${ }^{e}$ Danilo Kühn, ${ }^{a}$ Jerry LaRue, ${ }^{e, i}$ Giuseppe Mercurio, ${ }^{g}$ Michael P. Minitti ${ }^{f}$ Ankush Mitra, ${ }^{f}$ Stefan P. Moeller, ${ }^{f}$ May Ling Ng, ${ }^{e}$ Anders Nilsson, ${ }^{b, d, e}$ Dennis Nordlund, ${ }^{b}$ Jens Nørskov, ${ }^{e}$ Henrik Öström, ${ }^{d}$ Hirohito Ogasawara, ${ }^{b}$ Mats Persson, ${ }^{j}$ William F. Schlotter, ${ }^{f}$ Jonas A. Sellberg, ${ }^{b, d, k}$ Martin Wolf, Frank Abild-Pedersen, ${ }^{e}$ Lars G. M. Pettersson, ${ }^{d}$ Wilfried Wurth, ${ }^{c, g}$

${ }^{\text {a }}$ Institute for Methods and Instrumentation in Synchrotron Radiation Research, HelmholtzZentrum Berlin für Materialien und Energie GmbH, 12489 Berlin, Germany

${ }^{\mathrm{b}}$ SSRL, SLAC National Accelerator Laboratory, Menlo Park, CA 94025, USA

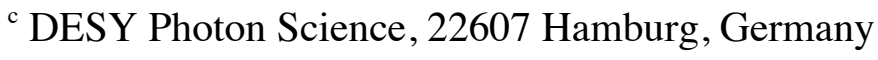

${ }^{d}$ Department of Physics, Stockholm University, 10691 Stockholm, Sweden

${ }^{\mathrm{e}}$ SUNCAT, SLAC National Accelerator Laboratory, Menlo Park, CA 94025, USA

${ }^{\mathrm{f}}$ LCLS, SLAC National Accelerator Laboratory, Menlo Park, CA 94025, USA

${ }^{g}$ Physik Department, Universität Hamburg and Center for Free-Electron Laser Science, 22761 Hamburg, Germany 


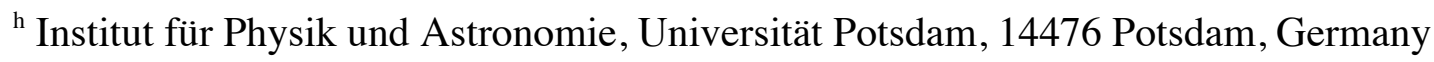

${ }^{i}$ Chapman University, Orange, CA 92866, USA

${ }^{\mathrm{j}}$ Surface Science Research Centre and Department of Chemistry, The University of Liverpool, Liverpool, UK

${ }^{\text {k }}$ Department of Applied Physics, KTH Royal Institute of Technology, 10691 Stockholm, Sweden

${ }^{1}$ Fritz-Haber-Institut der Max-Planck-Gesellschaft, 14195 Berlin, Germany

\section{Corresponding Author}

* E-Mail: martin.beye@desy.de 
ABSTRACT The concept of bonding and antibonding orbitals is fundamental in chemistry. The population of those orbitals and the energetic difference between the two reflect the strength of the bonding interaction. Weakening the bond is expected to reduce this energetic splitting, but the transient character of bond-activation has so far prohibited direct experimental access. Here we apply time-resolved soft X-ray spectroscopy at a free-electron laser to directly observe the decreased bonding-antibonding splitting following bond-activation using an ultrashort optical laser pulse.

\section{TOC GRAPHICS}

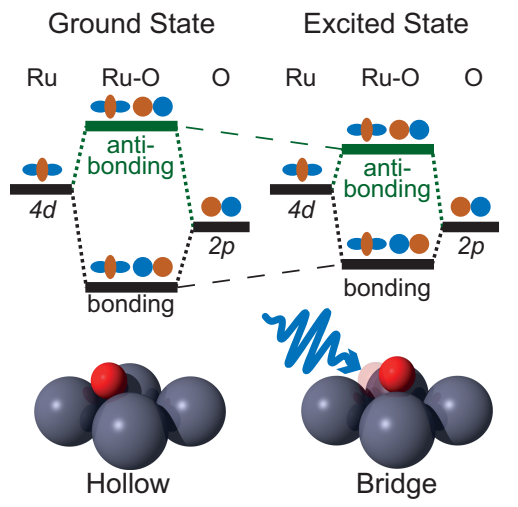


One of the most fundamental notions in chemistry is covalent bonding through formation of molecular orbitals via mixing of atomic orbitals. When each of two reaction partners contributes one orbital to the bond, the orbitals combine to two molecular orbitals, where one forms a bonding orbital which is shifted down in energy, while the other becomes antibonding and is shifted up in energy. When the latter orbital has a smaller occupation than the bonding orbital, the bond is stabilized. The energetic bond strength can be directly related to the resulting energetic splitting of the molecular orbitals and their occupancy.

Here we study how the bonding-antibonding splitting changes upon bond activation in a model system for heterogeneous catalysis: atomic oxygen chemisorbed on a ruthenium metal surface ${ }^{1,2}$. We use a femtosecond optical laser pulse to trigger a sequence of events where part of the excited metal electrons get transiently promoted into unoccupied states that are antibonding between oxygen and the surface. This weakens the bond, which leads to bond-elongation and a reduced bonding-antibonding splitting in the now activated system ${ }^{3,4}$. We directly follow the weakening of the oxygen to surface bond by monitoring the evolution of the energetic position of the bonding and antibonding states with time-resolved soft X-ray spectroscopy at a free-electron $\mathrm{X}$-ray laser ${ }^{5-10}$. The unoccupied antibonding states are measured with oxygen $K$-edge $\mathrm{X}$-ray absorption spectroscopy (XAS), while the occupied bonding states are analyzed by (resonant) Xray emission spectroscopy (XES). 


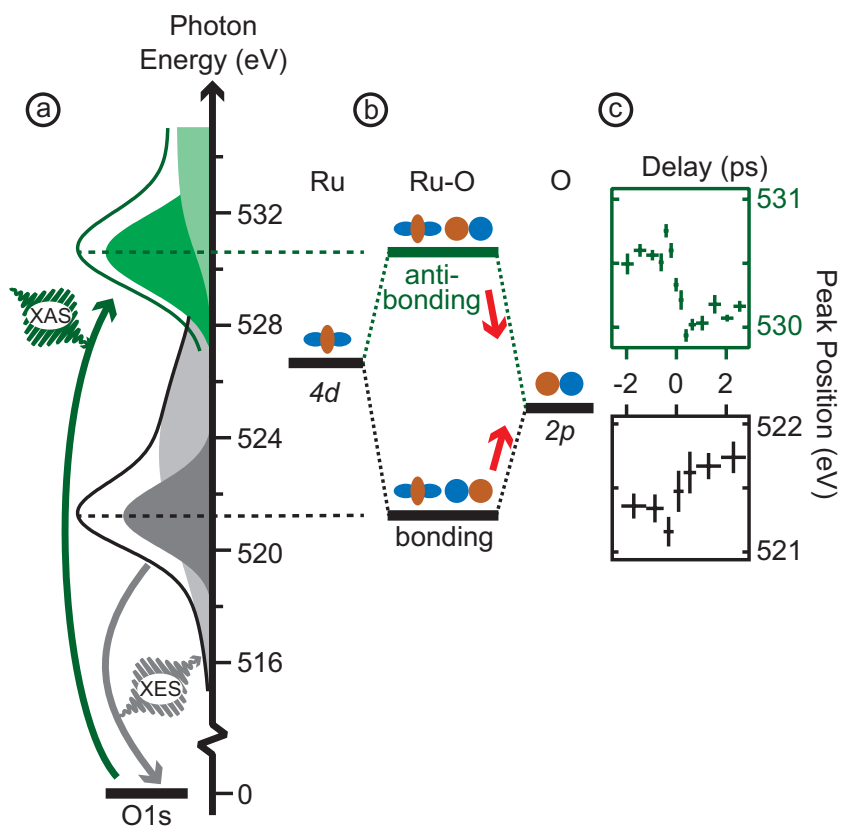

Figure 1. (a): Electronic transitions for core level spectroscopy. Top: The resonant absorption of an X-ray photon at the oxygen $1 s$ core level probes the unoccupied, antibonding states (green). Bottom: X-ray emission from the core-excited states represents the occupied $p$-density of states, mainly of bonding character.

(b): Sketch of orbital mixing of oxygen on ruthenium. When oxygen approaches the ruthenium surface, the $\mathrm{Ru} 4 d$ states mix with $\mathrm{O} 2 p$ levels to form bonding and antibonding orbitals (illustrated with $4 d_{\sigma}$ and $2 p_{z}$, the mixing with $5 s$ states is not shown). Their energetic splitting is related to the bond strength.

(c): Temporal evolution of the orbital binding energies. Promptly after laser excitation, the bond weakening is observed through a reduction of the bonding-antibonding splitting. The unoccupied antibonding orbitals (green) shift to lower energy while the occupied bonding states shift to higher photon energies upon laser excitation. 
In Figure 1.a, we show a sketch of how the X-ray transitions allow studying the splitting between the bonding and the antibonding orbitals ${ }^{11}$ : In X-ray absorption (green), an electron from the oxygen $1 s$ core level is excited to an unoccupied state determined by the incident photon energy; XAS thus measures the unoccupied states which here are mainly the O-Ru antibonding states. In XES (black), we study the radiative decay of this core-excited state. The $\mathrm{XE}$ spectrum is directly recorded with the spectrometer and provides a measure of the occupied p-density of states; here we will focus on the O-Ru bonding state.

Figure 1.b illustrates the orbital mixing when oxygen approaches the surface: the atomic valence $2 p$ orbitals interact with the $\mathrm{Ru} 4 d$ states and form two sets of molecular orbitals separated in energy. They can be classified as bonding and antibonding. (The interaction of oxygen orbitals with the broadband $5 s$ states in $\mathrm{Ru}$ is not shown.) Fig. 1.c presents the ultrafast reduction of their energetic splitting after exciting the system.

In the ground state, oxygen atoms on a ruthenium surface bind to a three-fold coordinated hollow site. A multitude of electronic states distributed over several electronvolts in energy is formed through intermixing the different orbitals (calculations are presented in Fig. 2.c). Here we use the term orbitals (strictly often used for small molecules only) to identify that the electronic states carry some adsorbate character. Two different sets of states can be observed ${ }^{2}$ : On one hand, states that are spread in energy over several electronvolts above and below the Fermi level with a constantly low density of states are derived from the interaction of the oxygen $2 p$ level with the broad $\mathrm{Ru} 5 s$ band. On the other hand, the interaction with well-localized $\mathrm{Ru} 4 d$ states yields two prominent features dominating the electronic structure: one peak with a width of 1-2 $\mathrm{eV}$ at lower binding energy which is completely occupied and one $2-3 \mathrm{eV}$ wide peak which is at 
higher energy and completely unoccupied. Those two features can be directly related to the simple concept of bonding and antibonding molecular orbitals.

In Figure 2.a, we display in detail the electronic structure of atomic oxygen adsorbed on a ruthenium surface as measured with our spectroscopy before the surface is excited. Only the bonding orbitals are fully occupied and appear in XES (black), while the antibonding orbitals appearing in XAS (green) are unoccupied. Together with the large energetic splitting, this results in a strong chemisorption bond (see also the supplement).

Figure 2.b shows how the spectra change during the first picoseconds after laser excitation. For comparison, the fits of the spectra at negative delay (i.e. the static initial state as in Fig. 2.a) are shown as dashed lines. After photoexcitation the bonding state in the XE spectrum has shifted to higher energy and lost peak intensity. An increased broadening of the peak is barely visible but is extracted in a fit. The antibonding state in the XA spectrum shifts to lower energy and is more obviously broadened.

From the fits, we extract a maximum shift of the position of the XA antibonding resonance by about $-0.56 \pm 0.07 \mathrm{eV}$ instantly after excitation only limited by the time resolution. The XE bonding resonance shifts by about $+0.37 \pm 0.11 \mathrm{eV}$. Simultaneously, the width of the antibonding peak increases by about $15 \%$. These shifts directly indicate a bond weakening represented by a decrease in the bonding-antibonding splitting of about $-0.93 \pm 0.18 \mathrm{eV}$. We note that on a timescale of about 10 ps after the laser excitation (see the supplement), the splitting is nearly restored to its original value, i.e. the oxygen is only transiently activated here.

In Figure 2.c, a simulation of the spectra is displayed for the initial state (dashed) and for the bond weakened state, where the oxygen atom is assumed to be promoted from the strongly binding hollow site to a bridge site with a larger oxygen to metal distance; the chosen 
configurations correspond to the initial and transition state in an oxygen diffusion simulation between adjacent hollow sites. The simulation reproduces the overall trends in reducing the energetic splitting between the bonding and antibonding states, the broadening and decrease of the peak heights. The computed spectral changes for this model are larger than observed in the experiment, indicating that in the experiment not all oxygen atoms move completely to the bridge site. The experimental spectra are additionally broadened as a result of ensemble and temporal averaging as well as due to vibrational excitations that are not included in the calculation.

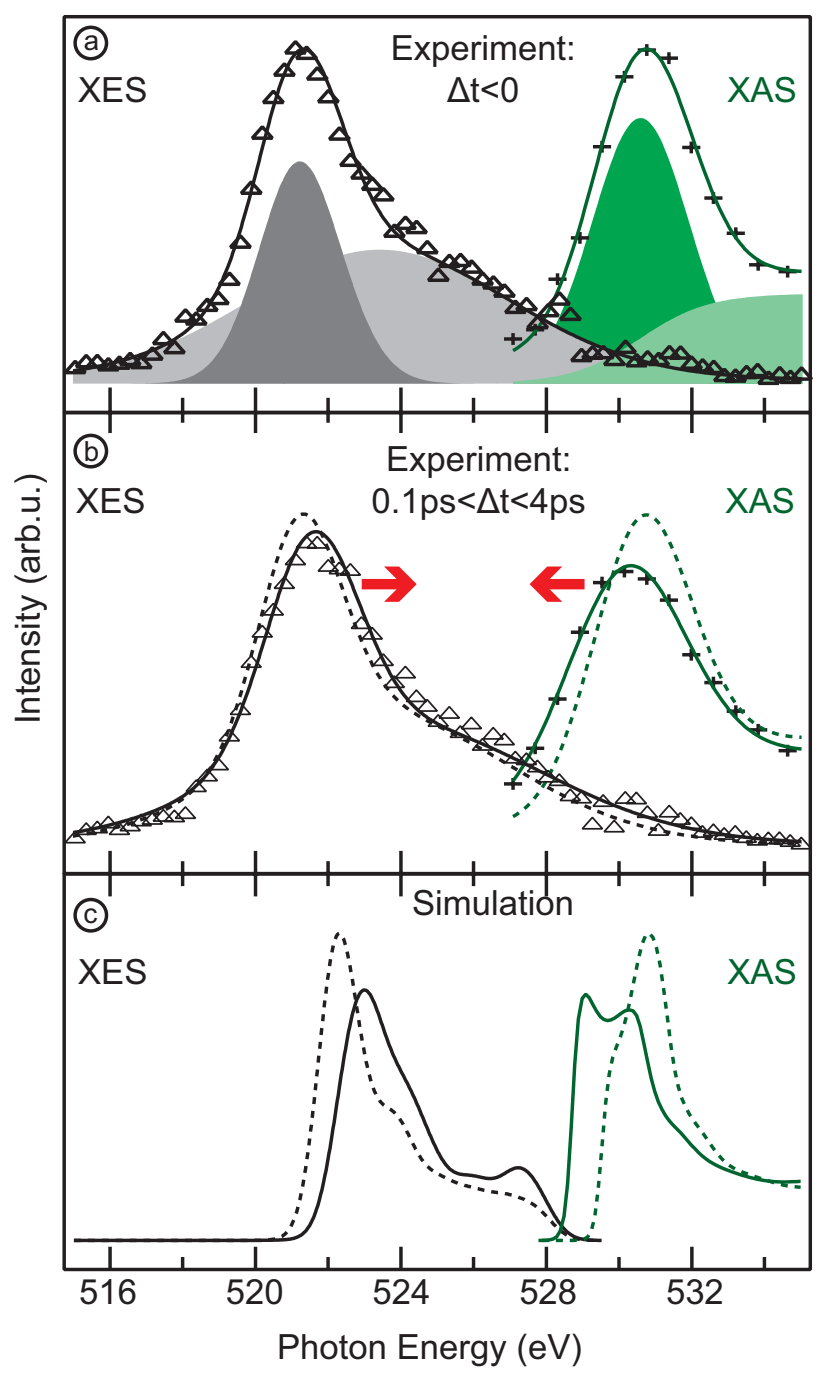


Figure 2. (a) Contributions to the ground state spectra. Before the laser excitation, the bonding occupied states (grey, XES) and antibonding unoccupied states (green, XAS) are decomposed into the respective contributions. (The elastic scattering peak has been removed in panels (a) and (b) to ease comparison with the simulations. See also Figure 3 in the Experimental methods section.)

(b) Spectral evolution after optical pumping. After the laser excitation, the splitting between the bonding and antibonding states is decreased, indicative of a weakened bond. Negative delay spectra are overlaid as dashed lines for comparison.

(c) Spectrum simulation for the tightly bound initial state (dashed) of oxygen in a hollow site of the ruthenium surface and for the bridge site with the oxygen in-between two hollow sites (solid) with a weakened bond.

To interpret these spectral changes in detail, we discuss the excitation mechanism and the basic processes on a surface after ultrashort laser excitation ${ }^{12}$ : Optical absorption occurs almost exclusively in the metal while direct photo excitation of the adsorbate is negligible. Thereby valence electrons in the metal are excited into the conduction band and in less than 100 fs thermalize towards a hot electron gas with a temperature of around $6000 \mathrm{~K}$ for the conditions in our experiment ${ }^{13}$.

On the same 100 fs timescale, the excited electron distribution indirectly induces strong adsorbate vibrations via the following mechanism ${ }^{4}$ : Temporarily, excited electrons from the metal can occupy substrate-adsorbate repulsive antibonding states ${ }^{3}$. This weakens the bond that consequently starts to elongate. The oxygen atoms begin to move away from the strongly bound 
hollow site. When the electrons transfer back to the substrate, the oxygen atoms are left in a vibrationally excited state with an average position towards the bridge site. As a stochastic process, this contributes to the spectral broadening. Nevertheless, the largest excursion of the oxygen atoms towards the bridge site is reached within our time resolution of about 190 fs. The substrate-adsorbate bond at the bridge site is weaker and this bond weakening is the main cause for the observed spectral changes - the decreased bonding-antibonding splitting as direct evidence of the weakened interaction between adsorbate and surface.

In conclusion, we demonstrate here directly in the electronic structure, how the (transient) population of antibonding states induces ultrafast geometrical changes, which in turn result in a weakened bond and consequently a reduced splitting between bonding and antibonding orbitals. This constitutes a direct experimental demonstration of the fundamental concept of bonding and antibonding orbitals and their relation to the chemical bond strength, which can directly connect to the activation of a bond for reactions ${ }^{8}$.

\section{Experimental Methods}

Measurements: The experiments were performed at the soft X-ray materials science (SXR) beamline at the Linac Coherent Light Source (LCLS) described in reference ${ }^{14}$. We used the surface science endstation (SSE) equipped with a home-built X-ray emission spectrometer using the setup as presented in detail in reference ${ }^{9}$. The background pressure was in the $10^{-10}$ Torr range. We cleaned a Ru (0001) single-crystal through sputtering and annealing with and without oxygen. An initial $\mathrm{p}(2 \mathrm{x} 1)$ layer of oxygen was prepared by exposing the clean crystal to oxygen at about room temperature according to the recipe from Lizzit et al. ${ }^{15}$. The sample was placed at a grazing incidence angle of approximately $2^{\circ}$ into the soft X-ray beam and the collinear $400 \mathrm{~nm}$ 
pump laser beam both at $120 \mathrm{~Hz}$ and focused to a spot size of about $50 \mu \mathrm{m}$ by $50 \mu \mathrm{m}$. It was ensured that no sample damage was involved by comparing spectra recorded over several minutes on the same spot. No differences were found, but as a precaution, the sample was scanned during the measurement. LCLS was operated with 80 fs long electron bunches, while the central photon energy was scanned around the $\mathrm{O} 1 \mathrm{~s}$ edge with the beamline monochromator set to a resolution of $250 \mathrm{meV}$. The optical laser was $p$-polarized to the sample at a fluence of approximately $140 \mathrm{~J} / \mathrm{m}^{2}$. Temporal jitter between both pulses was monitored shot to shot and corrected for during data analysis using a spatially encoding cross-correlator as described in reference ${ }^{16}$. Ultimately, the length of the optical laser pulses of about 170 fs limited the time resolution to $190 \mathrm{fs}$.

XE spectra of oxygen adsorbed on ruthenium do not show a dependence on the excitation energy besides a strong overall intensity scaling with the XA profile within the current energy resolution. This is characteristic for well-screened states in metals and here the chemisorbed oxygen on a metal behaves as in a metallic state ${ }^{17}$. For the displayed XE spectra, the spectrometer counts for the excitation energy range between 529.5 and $530.4 \mathrm{eV}$ were collected. This range was chosen, since this is where the largest spectral changes are observed in XAS. We thus enhance the XE signal from excited atoms that show a shifted XAS resonance ${ }^{7}$. When recording emission spectra, a contribution from elastic scattering at the excitation energy is always observed. The related peak has been fitted and removed from Figure 2. For completeness, in Figure 3, we show the XE spectra as recorded.

The XA spectra have been obtained by summing all the spectrometer counts for emission energies between 517 and $537 \mathrm{eV}$. Note that the intensity scaling between the XE and XA spectra is arbitrary and has been chosen such that the initial state peak heights match. 
Absolute splitting and screening response: We note that we cannot directly extract the absolute value of the bonding-antibonding splitting from our experimental spectra, but relative changes can still be accurately extracted. This is due to a constant offset mainly affecting the absorption spectra. It results from the $1 s$ core hole in the final state of the absorption process triggering a screening reaction of valence electrons that lowers the total energy of the system and thus the XAS energy ${ }^{18,19}$. The screening energy can be included in theoretical calculations and mainly depends on the number of screening valence electrons while being independent from their energetic distribution. The laser excitation thus does not alter the screening response, allowing us to extract relative shifts of the spectra that can be directly related to the change of the bondingantibonding splitting.

Fit model: The XE spectra have been fitted with an empirical model consisting of three Gaussian peak functions (shaded grey). The peaks represent the main occupied oxygen valence states (at $521 \mathrm{eV}$ ), a broad feature from the hybridization of broadband ruthenium states with oxygen orbitals and a peak (around $531 \mathrm{eV}$ ) resulting from elastic scattering. This latter peak provides the energy calibration of the spectrometer to the incoming photon energy and has been removed from the figure in the main paper (compare Figure 3). The ground state model parameters have been determined from an unconstrained fit. In order to systematically track only significant pump-probe delay dependent changes, the set of free fit parameters has been reduced as far as possible without compromising on fit quality. It has been found that the broad feature changes in all parameters together with the main peak. Position, height and width are thus fixed relative to the main peak for the delay dependent fits. 
The XA spectra have been fitted with a Gaussian function, representing the unoccupied oxygen states and a hyperbolic tangent function to model the continuum edge jump (shaded light green in Figures 1.a and 2.a).

The XA and XE spectrum fits have been performed for each time step in the delay scan (see Figure 4). Error bars are given as one standard deviation from the fit for values on the vertical axis, while the error bars on the delay axis represent the bin size.

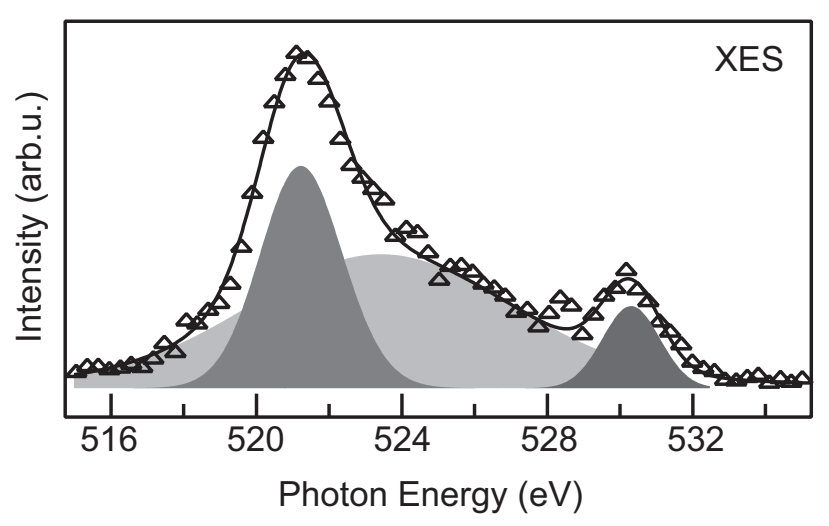

Figure 3: XE spectrum including the elastic peak. Recorded XE spectra always contain a contribution from elastic scattering appearing at the excitation energy. The elastic peak (here visible around $530 \mathrm{eV}$ ) contains no further electronic structure information. Here it has been fitted together with the rest of the spectrum. For better visualization of the occupied states as measured with XE, the elastic peak has been subtracted in Figure 2. Here, as an example, we show the ground state spectrum as measured containing the elastic peak. 


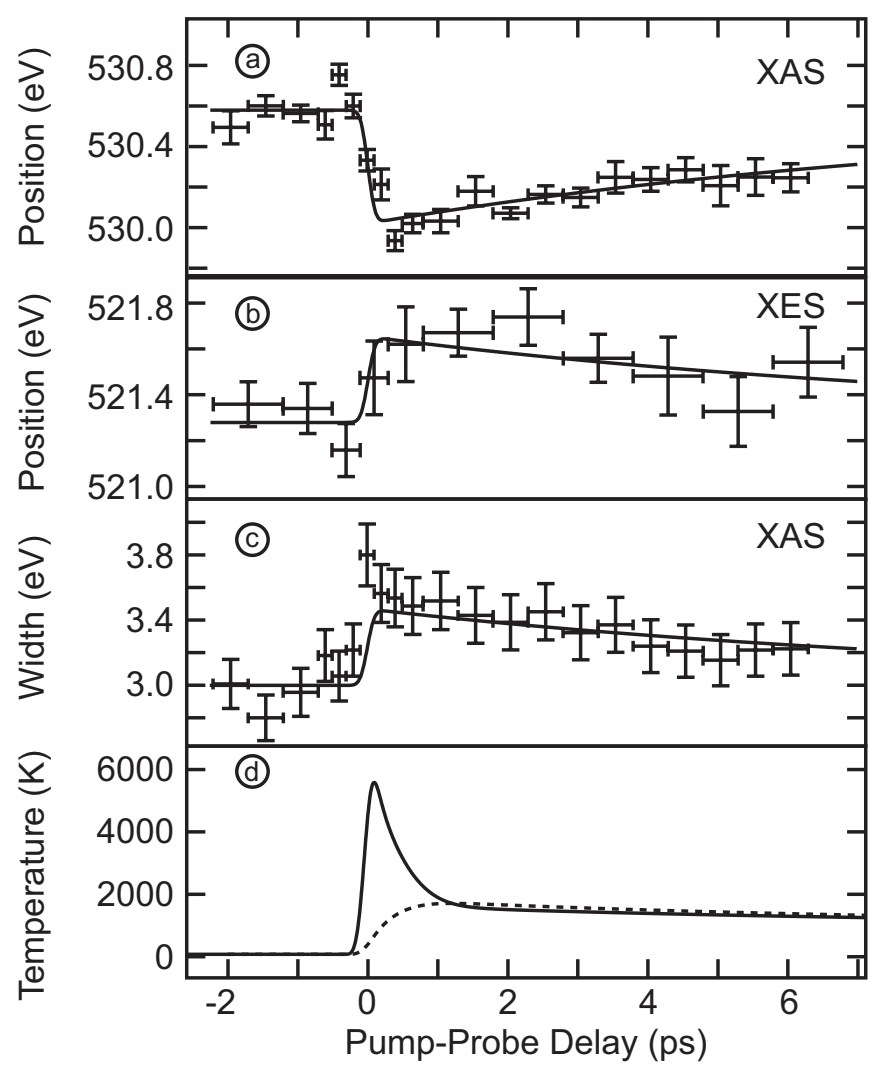

Figure 4: (a) Fitted position of the peak in XAS.

(b) Fitted position of the peak in XES. As compared to XAS, the statistical quality is lower, since the same measured signal is not spectrally analysed. Bin size and error bars are thus larger.

(c) Fitted width of the XAS peak. The increase in width is mainly a result of vibrational excitations and ensemble averaging.

The solid curves in (a)-(c) result from a global fit of the excitation and decay time constants. The excitation time constant is compatible with a ultrafast excitation within the 190 fs temporal resolution. The single exponential decay time constant is fitted to $9.5 \pm 2$ ps.

(d) The solid line displays the temperature evolution of the electrons (phonon temperature is dashed) resulting from a calculation using the two-temperature model with parameters from ${ }^{6}$ and references therein. 


\section{Computational Methods}

Two-temperature calculations performed with parameters from ${ }^{6}$ (see also references therein) for the substrate electron and phonon temperature reveal a rather quick equilibration towards $2000 \mathrm{~K}$ within the first picoseconds after laser excitation (see Figure 4). For this substrate temperature using the Climbing Image-Nudged Elastic Band (CI-NEB) method ${ }^{20}$ the minimum free energy path was calculated from the hcp hollow via the bridge site to the fcc hollow site. An entropic correction was included but turned out to be less than $60 \mathrm{meV}$. The potential of mean force was calculated following Tully et al. ${ }^{21,22}$ by integrating all degrees of freedom orthogonal to the pathway. A free energy barrier of about $0.7 \mathrm{eV}$ located at the bridge site was found.

Spectra were calculated for the initial ground state structure with the oxygen atoms located at the hcp hollow site, as well as for the bridge site structure extracted from the free energy simulations. The GPAW code was used ${ }^{23}$. A $2 \times 2$ slab of four atomic layers representing the Ru surface and a $4 \times 4 \times 1 k$-point sampling was employed to sample the Brillouin zone. XE spectra were obtained using frozen orbitals ${ }^{24}$ and broadened using a Gaussian function with a full width at half maximum of $1.0 \mathrm{eV}$. XA spectra were calculated using the transition state potential method ${ }^{25}$. The unoccupied states were constructed using a Haydock recursion scheme with 2000 Lanczos vectors ${ }^{26}$. To avoid core-hole interactions between unit cells in the XA calculations, the cells were enlarged accordingly.

\section{AUTHOR INFORMATION}

\section{Corresponding Author}

E-Mail (Martin Beye): martin.beye@desy.de

\section{Notes}


The authors declare no competing financial interests.

\section{ACKNOWLEDGMENT}

This research was carried out on the SXR Instrument at the Linac Coherent Light Source (LCLS) at the SLAC National Accelerator Laboratory. The SXR Instrument is funded by a consortium whose membership include the LCLS, Stanford University through the Stanford Institute for Materials Energy Sciences (SIMES), Lawrence Berkeley National Laboratory (LBNL), University of Hamburg through the BMBF priority program FSP 301, and the Center for Free Electron Laser Science (CFEL). The LCLS is funded by the DOE Office of Basic Energy Sciences. The spectrum calculations were performed on resources provided by the Swedish National Infrastructure for Computing (SNIC) at the HPC2N center. We gratefully acknowledge the support of the LCLS staff. This work was further supported by the Swedish research council (VR), the Alexander von Humboldt foundation, the DFG within the excellence cluster "Center for Ultrafast Imaging (CUI)", by the US Department of Energy, Basic Energy Science through the SUNCAT Center for Interface Science and Catalysis and the VolkswagenStiftung.

\section{REFERENCES}

(1) Zhang, C. J.; Hu, P. Why Must Oxygen Atoms Be Activated From Hollow Sites to Bridge Sites in Catalytic CO Oxidation? J Am Chem Soc 2000, 122, 2134-2135.

(2) Nørskov, J. K.; Studt, F.; Abild-Pedersen, F.; Bligaard, T. Fundamental Concepts in Heterogeneous Catalysis; Wiley: Hoboken, NJ; 2014. 
(3) Bonn, M.; Funk, S.; Hess, C.; Denzler, D. N.; Stampfl, C.; Scheffler, M.; Wolf, M.; Ertl, G. Phonon- Versus Electron-Mediated Desorption and Oxidation of $\mathrm{CO}$ on $\mathrm{Ru}(0001)$. Science 1999, 285, 1042-1045.

(4) Öberg, H.; Gladh, J.; Marks, K.; Ogasawara, H.; Nilsson, A. R.; Pettersson, L. G. M.; Öström, H. Indication of Non-Thermal Contribution to Visible Femtosecond Laser-Induced CO Oxidation on $\mathrm{Ru}(0001)$. J. Chem. Phys. 2015, 143, 074701.

(5) Nilsson, A. R.; Pettersson, L. G. M. Chemical Bonding on Surfaces Probed by X-Ray Emission Spectroscopy and Density Functional Theory. Surf. Sci.Rep. 2004, 55, 49-167.

(6) Dell'Angela, M.; Anniyev, T.; Beye, M.; Coffee, R.; Föhlisch, A.; Gladh, J.; Katayama, T.; Kaya, S.; Krupin, O.; LaRue, J.; et al. Real-Time Observation of Surface Bond Breaking with an X-Ray Laser. Science 2013, 339, 1302-1305.

(7) Beye, M.; Anniyev, T.; Coffee, R.; Dell'Angela, M.; Föhlisch, A.; Gladh, J.; Katayama, T.; Kaya, S.; Krupin, O.; Mogelhoj, A.; et al. Selective Ultrafast Probing of Transient Hot Chemisorbed and Precursor States of CO on Ru(0001). Phys. Rev. Lett. 2013, 110, 186101.

(8) Öström, H.; Öberg, H.; Xin, H.; LaRue, J.; Beye, M.; Dell'Angela, M.; Gladh, J.; Ng, M. L.; Sellberg, J. A.; Kaya, S.; et al. Probing the Transition State Region in Catalytic CO Oxidation on Ru. Science 2015, 347, 978.

(9) Katayama, T.; Anniyev, T.; Beye, M.; Coffee, R.; Dell'Angela, M.; Föhlisch, A.; Gladh, J.; Kaya, S.; Krupin, O.; Nilsson, A. R.; et al. Ultrafast Soft X-Ray Emission Spectroscopy of Surface Adsorbates Using an X-Ray Free Electron Laser. J. Electron Spectrosc. Relat. Phenom. 2013, 187, 9-14. 
(10) Chergui, M. Time-Resolved X-Ray Spectroscopies of Chemical Systems: New Perspectives. Struct. Dyn. 2016, 3, 031001-031013.

(11) Wiell, T.; Klepeis, J. E.; Bennich, P.; Björneholm, O.; Wassdahl, N.; Nilsson, A. R. Local Aspects of the Adsorbate-Substrate Chemical Bond in $\mathrm{N} / \mathrm{Cu}(100)$ and $\mathrm{O} / \mathrm{Cu}(100)$. Phys. Rev. B 1998, 58, 1655 .

(12) Frischkorn, C.; Wolf, M. Femtochemistry at Metal Surfaces: Nonadiabatic Reaction Dynamics. Chem Rev 2006, 106, 4207-4233.

(13) Lisowski, M.; Loukakos, P.; Bovensiepen, U.; Staehler, J.; Gahl, C.; Wolf, M. Ultra-Fast Dynamics of Electron Thermalization, Cooling and Transport Effects in Ru(001). Appl. Phys. A Mater. 2004, 78, 165-176.

(14) Schlotter, W. F.; Turner, J. J.; Rowen, M.; Heimann, P. A.; Holmes, M.; Krupin, O.; Messerschmidt, M.; Moeller, S.; Krzywinski, J.; Soufli, R.; et al. The Soft X-Ray Instrument for Materials Studies at the Linac Coherent Light Source X-Ray Free-Electron Laser. Rev. Sci. Instrum. 2012, 83, 043107.

(15) Lizzit, S.; Baraldi, A.; Groso, A.; Reuter, K.; Ganduglia-Pirovano, M. V.; Stampl, C.; Scheffler, M.; Stichler, M.; Keller, C.; Wurth, W.; et al. Surface Core-Level Shifts of Clean and Oxygen-Covered Ru(0001). Phys. Rev. B 2001, 63, 205419.

(16) Beye, M.; Krupin, O.; Hays, G.; Reid, A. H.; Rupp, D.; de Jong, S.; Lee, S.; Lee, W.-S.; Chuang, Y. D.; Coffee, R.; et al. X-Ray Pulse Preserving Single-Shot Optical Cross-Correlation Method for Improved Experimental Temporal Resolution. Appl. Phys. Lett. 2012, 100, 121108. 
(17) Nilsson, A.; Pettersson, L. G. M. Chemical Bonding on Surfaces Probed by X-ray Emission Spectroscopy and Density Functional Theory. Surf. Sci.Rep. 2004, 55, 49-167.

(18) Stöhr, J. NEXAFS Spectroscopy; Springer Series in Surface Sciences; Springer Berlin Heidelberg: Berlin, Heidelberg; 1992.

(19) Miedema, P. S.; Beye, M.; Könnecke, R.; Schiwietz, G.; Föhlisch, A. Thermal Evolution of the Band Edges of 6H-SiC: X-Ray Methods Compared to the Optical Band Gap. J Electron Spectrosc 2014, 197, 37-42.

(20) Henkelman, G.; Uberuaga, B. P.; Jónsson, H. A Climbing Image Nudged Elastic Band Method for Finding Saddle Points and Minimum Energy Paths. J. Chem. Phys. 2000, 113, 9901.

(21) Doren, D. J.; Tully, J. Precursor-Mediated Adsorption and Desorption - a TheoreticalAnalysis. Langmuir 1988, 4, 256-268.

(22) Doren, D. J.; Tully, J. C. Dynamics of Precursor-Mediated Chemisorption. J. Chem. Phys. 1991, 94, 8428.

(23) Enkovaara, J.; Rostgaard, C.; Mortensen, J. J.; Chen, J.; Dułak, M.; Ferrighi, L.; Gavnholt, J.; Glinsvad, C.; Haikola, V.; Hansen, H. A.; et al. Electronic Structure Calculations with GPAW: a Real-Space Implementation of the Projector Augmented-Wave Method. J. Phys.: Condens. Matter 2010, 22, 253202.

(24) Triguero, L.; Pettersson, L. G. M.; Ågren, H. Calculations of X-Ray Emission Spectra of Molecules and Surface Adsorbates by Means of Density Functional Theory. J. Phys. Chem. A 1998, 102, 10599-10607. 
(25) Triguero, L.; Pettersson, L. G. M.; Ågren, H. Calculations of Near-Edge X-RayAbsorption Spectra of Gas-Phase and Chemisorbed Molecules by Means of Density-Functional and Transition-Potential Theory. Phys. Rev. B 1998, 58, 8097.

(26) Ljungberg, M. P.; Mortensen, J. J.; Pettersson, L. G. M. An Implementation of Core Level Spectroscopies in a Real Space Projector Augmented Wave Density Functional Theory Code. J. Electron Spectrosc. Relat. Phenom. 2011, 184, 427-439. 